\title{
Enchanted by Your Surrounding? Measuring the Effects of Immersion and Design of Virtual Environments on Decision-Making
}

\author{
Sebastian Oberdörfer ${ }^{1 *}$, David Heidrich ${ }^{2}$, Sandra Birnstiel ${ }^{1}$ and Marc Erich Latoschik ${ }^{1}$ \\ ${ }^{1}$ Chair for Human-Computer Interaction, University of Würzburg, Würzburg, Germany, ${ }^{2}$ Institute for Software Technology, \\ German Aerospace Center (DLR), Weßling, Germany
}

Impaired decision-making leads to the inability to distinguish between advantageous and disadvantageous choices. The impairment of a person's decision-making is a common goal of gambling games. Given the recent trend of gambling using immersive Virtual Reality it is crucial to investigate the effects of both immersion and the virtual environment (VE) on decision-making. In a novel user study, we measured decision-making using three virtual

OPEN ACCESS

Edited by:

Missie Smith,

Independent researcher,

United States

Reviewed by:

Phil Lopes,

Universidade Lusófona, Portugal

Robert Gutzwiller,

Arizona State University, United States

*Correspondence:

Sebastian Oberdörfer sebastian.oberdoerfer@uniwuerzburg.de

Specialty section: This article was submitted to Virtual Reality and Human Behaviour, a section of the journal

Frontiers in Virtual Reality

Received: 11 March 2021 Accepted: 20 July 2021

Published: 06 August 2021

Citation: Oberdörfer S, Heidrich D, Birnstiel S and Latoschik ME (2021) Enchanted by Your Surrounding? Measuring the Effects of Immersion and Design of Virtual Environments on Decision-

Making

Front. Virtual Real. 2:679277. doi: 10.3389/frvir.2021.679277 versions of the lowa Gambling Task (IGT). The versions differed with regard to the degree of immersion and design of the virtual environment. While emotions affect decisionmaking, we further measured the positive and negative affect of participants. A higher visual angle on a stimulus leads to an increased emotional response. Thus, we kept the visual angle on the lowa Gambling Task the same between our conditions. Our results revealed no significant impact of immersion or the VE on the IGT. We further found no significant difference between the conditions with regard to positive and negative affect. This suggests that neither the medium used nor the design of the VE causes an impairment of decision-making. However, in combination with a recent study, we provide first evidence that a higher visual angle on the IGT leads to an effect of impairment.

Keywords: virtual reality, virtual environments, immersion, decision-making, iowa gambling task

\section{INTRODUCTION}

Real-life decision-making situations are complex. People must deal with uncertainties in the context of punishment and reward (Brevers et al., 2013). While experiencing an impaired decision-making, people might purposefully make decisions that seem beneficial to them despite being clearly disadvantageous. This also applies to artificial situations, such as training (Leder et al., 2019), gaming (Oberdörfer and Latoschik, 2013; Oberdörfer and Latoschik, 2018), and gambling (Griffiths, 2017) in Virtual Reality (VR). In the latter example, impaired decision-making can lead to an attempt to compensate for a substantial loss by making even more risky decisions in the next moves (Gainsbury et al., 2014). A recent study revealed a higher risk potential of gambling games when played in immersive VR (Heidrich et al., 2019).

Research has measured and analyzed decision-making using the Iowa Gambling Task (IGT) (Bechara et al., 1994; Brevers et al., 2013) for more than 20 years. The task simulates real-life decision-making featuring uncertainties with respect to assumptions and outcomes. During the task, participants draw cards from four different decks. In the long run, two of these decks are advantageous and two are disadvantageous. Typically, the number of advantageous minus 

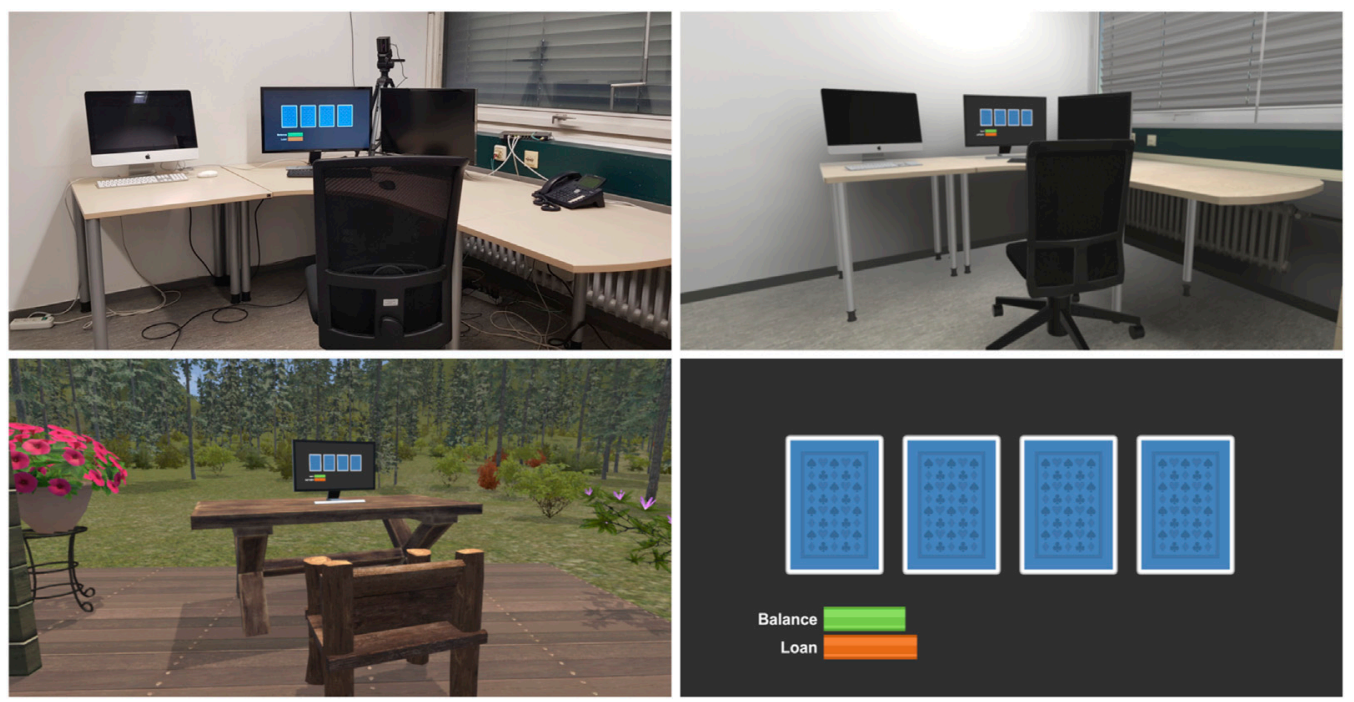

FIGURE 1 | Comparison of our three IGT versions. Top left: IGT played on desktop in the laboratory. Top right: IGT played in the virtual laboratory. Bottom left: IGT played in the virtual forest. Bottom right: IGT user interface for all versions.

disadvantageous cards drawn determines a subject's decisionmaking. In addition, analyzing the decision-making patterns over the course of the task provides further insights into the cognitive and emotional processes (Bechara et al., 2002; Bowman et al., 2005; van den Bos et al., 2006). In a previous study, we found an impairment of decision-making when completing the IGT in immersive VR (Oberdörfer et al., 2020). Our first realization of IGT VR directly integrated the task into a virtual environment (VE). As a result, the two conditions, desktop-3D and immersive VR, not only differed in the medium used, but also in the visual angle on the stimulus. Using immersive VR instead of a computer screen increases the visual angle on the VE and hence on the stimulus (Slater and Wilbur, 1997). A higher visual angle leads to an increased emotional response to audiovisual stimuli (Gall and Latoschik, 2020). As emotions can influence decision-making (Bechara and Damasio, 2005), we hypothesized that this higher visual angle was the true cause for an impairment of decisionmaking (Oberdörfer et al., 2020). Additionally, our VE was colorful, happy, and featured a high visual fidelity, i.e., an aquarium scenario. This might have influenced a player's emotions even further and hence increased the impairing effect.

Therefore, in alignment with the long tradition of determining the effect of immersion on human factors, e.g., on emotion and cognition (Visch et al., 2010), it is crucial to further investigate the impairment of decision-making in immersive VR by testing our hypothesis. This requires a twofold approach. First, the IGT needs to be administered on desktop and in VR while keeping the visual angle on the task the same. Second, the IGT needs to be integrated in VEs that differ with respect to their emotional design. These two experiments would facilitate an investigation whether 1) immersion or the visual angle on the task causes an impairing effect and 2) the surrounding VE influences decision-making.

\section{Contribution}

This article expands the research on the effects of immersion on decision-making. Following the identified research gaps, we developed three virtual IGT versions. The versions provided the same visual angle on the task but differed with regard to either the degree of immersion, i.e., low immersion desktop or high immersion VR, or design of the VE. The environmental settings included our laboratory, a virtual replicate of the physical laboratory, and a virtual forest as shown in Figure 1. In a novel user study, we measured IGT decision-making as well as positive and negative affect using the three versions. Comparing our results, we found no significant impact of immersion or the environment on IGT decision-making. We further found no significant difference between the conditions with regard to positive and negative affect. In combination with our previous IGT VR experiment (Oberdörfer et al., 2020), this provides first indications for a moderating effect of the visual angle on IGT decision-making. A higher visual angle increases emotional responses (Gall and Latoschik, 2020) and hence can directly affect decision-making. This is an important insight. It supports the advantage of using immersive VR for training of decision-making in critical situations. Also, it indicates the necessity to consider the visual angle on the game when assessing the risk of an immersive VR gambling game.

\section{THEORETICAL BACKGROUND}

The gambling industry continuously invests in new technologies to increase the attractiveness of gambling. To target young individuals, gambling is provided in immersive $V R$ (Griffiths, 2017). These new technologies might additionally increase the overall risk potential (Armstrong et al., 2017). A higher risk potential results in a higher chance to cause gambling related 
harm. Research already demonstrates an increased risk potential of a gambling game when played in immersive VR (Heidrich et al., 2019). Hence, it is crucial to investigate whether immersion influences other human factors, such as decision-making, that contribute to the risk of gambling in VR.

\section{1 lowa Gambling Task}

The IGT is an experimental paradigm for measuring real-life decision-making in a laboratory environment (Bechara et al., 1994; Betz et al., 2019). Commonly, the results are referred to as IGT decision-making. For more than 20 years, multiple areas of research used the IGT to measure and analyze decision-making and affecting factors (Chiu et al., 2018). For instance, induced time pressure (DeDonno and Demaree, 2008) and a participant's gender (van den Bos et al., 2013) and age (Cauffman et al., 2010) influenced IGT decision-making. Researchers further used the IGT to analyze the differences between controls groups and clinical populations such as cocaine users (Verdejo-Garcia et al., 2007) and pathological players (Brevers et al., 2012, 2013). More prominently, researchers administered the IGT to investigate the often underestimated effect of emotions on decision-making (Bechara and Damasio, 2005). For example, induced excitement showed an influence on the measurements (Preston et al., 2007; Miu et al., 2008). Researchers also administered the IGT to investigate the influence of emotions and mood evoked by sequences of movies on decision-making (de Vries et al., 2008; İyilikci and Amado, 2018). It was shown that a positive mood can lead to a better IGT decision-making in the first phase of the task (de Vries et al., 2008). Also, subjects in a certainty-associated emotional state outperformed those in an uncertainty-associated emotional state (İyilikci and Amado, 2018). Moreover, although not completely applicable, research demonstrated an influence on IGT decision-making of subjects suffering from anxieties when fear-relevant stimuli is displayed on either the advantageous or disadvantageous decks (Pittig et al., 2014). This is an important insight as it supports our research of investigating the effects of the surrounding VE on the IGT decision-making. The emotional design of a VE might influence IGT decision-making.

Subjects begin the IGT with a virtual loan of $\$ 2000$ and are tasked with maximizing their virtual money by drawing 100 cards from four different decks (Bechara et al., 1994). The decks are denoted as deck $A, B, C$, and $D$ and consist of 40 cards each in the original version of the IGT (Bechara et al., 1994). Each card has a concrete payout determined by a fixed win and loss schedule as displayed in Table 1. The value and the frequency of predetermined wins and losses result in decks A and B seeming advantageous in the short-term but being disadvantageous in the long run. In contrast, decks C and D seem disadvantageous in the short-term but are advantageous in the long run. Both advantageous decks lead to the same total profit and both disadvantageous decks lead to the same total loss as displayed in Table 2. This leads to a conception of risk in the form of intertemporality (Singh, 2013). However, with regard to the frequency of immediate reward and punishment, decks A and $\mathrm{C}$ could be perceived as more risky whereas decks $\mathrm{B}$ and $\mathrm{D}$ could be perceived as safe (Singh, 2013). Decks A and C feature a high frequency of small losses. In contrast, decks B and D include substantial losses at a low frequency. This results in the second conception of risk in the form of frequency. The perception of deck B being safe further can lead to the prominent deck $B$ phenomenon (Chiu et al., 2018). Subjects draw multiple cards from this deck. Participants receive no further information concerning the task itself, including the number of cards to be drawn, or the underlying principles such as the fixed schedule of wins and losses. As a result, the IGT simulates decision-making featuring an uncertainty in the context of punishment and reward (Brevers et al., 2013). The IGT showed to be robust in the face of certain changes in its parameters such as using it in the original 40-card manual version (Bechara et al., 1994), 60-card computerized version (Bechara et al., 2000), and computerized version with a higher value contrast (Lee et al., 2014).

The Somatic Marker Hypothesis (Damasio et al., 1991; Bechara and Damasio, 2005) has a high potential to explain this emotionally influenced IGT decision-making behavior (Brevers et al., 2013). According to this hypothesis, emotions can influence decision-making and cause a person to make unfavorable decisions. However, several studies demonstrated that the IGT can be completed with the development of as well as access to explicit knowledge and hence with cognitive processes (Maia and McClelland, 2004; Dunn et al., 2006). In addition, research demonstrated that healthy participants with a higher risk attitude purposefully draw cards from more risky decks (Singh and Khan, 2008). This results in a dichotomy of cognitionemotion in IGT decision-making (Kahneman and Frederick, 2007; Singh, 2013). Therefore, while emotions have been shown to constantly influence IGT decision-making (Heilman et al., 2010), they are not the only factor contributing to a subject's behavior on the task (Dunn et al., 2006). This could lead to emotions evoked by the rewards or punishments dominating the first phase of the IGT and the cognitive system taking over for the last phases of the task (Flores-Torres et al., 2019). This is in line with the observation that the decision time strongly declines over the course of the first two phases (Cella et al., 2007). In addition, a person's mood affects the IGT decision-making during the second phase of the task (de Vries et al., 2008). A positive mood leads to a better IGT decision-making.

An unimpaired subject completing the IGT typically experiences four phases, or periods of card selection (Bechara et al., 2005). These are the pre-punishment period, pre-hunch period, hunch period and conceptual period. Subjects begin with no knowledge about the distribution of advantageous and disadvantageous decks (Bechara et al., 1997). They usually prefer decks A and B during this pre-punishment period. Around the 10th game round, after experiencing a few losses, participants enter the pre-hunch period. Despite still not knowing which decks are advantageous or disadvantageous, they begin to develop a first hunch. Around the 50th game round, participants enter the hunch period. In this period, subjects begin to show minimal knowledge about the distribution of good and bad decks. This leads to a more pronounced decline in the number of disadvantageous cards drawn. Finally, participants enter the conceptual period by game round 80 . This period is associated with the development of knowledge about the underlying 
TABLE 1 | Overview of the decks and their win and loss schedule. Values are in $\$$.

\begin{tabular}{|c|c|c|c|c|c|c|c|c|c|c|c|c|c|c|c|c|c|c|c|c|}
\hline Number of Card & 1 & 2 & 3 & 4 & 5 & 6 & 7 & 8 & 9 & 10 & 11 & 12 & 13 & 14 & 15 & 16 & 17 & 18 & 19 & 20 \\
\hline \multicolumn{21}{|l|}{ Deck A } \\
\hline Win & +100 & +100 & +100 & +100 & +100 & +100 & +100 & +100 & +100 & +100 & +100 & +100 & +100 & +100 & +100 & +100 & +100 & +100 & +100 & +100 \\
\hline Loss & 0 & 0 & -150 & 0 & -300 & 0 & -200 & 0 & -250 & -350 & 0 & -350 & 0 & -250 & -200 & 0 & -300 & -150 & 0 & 0 \\
\hline \multicolumn{21}{|l|}{ Deck B } \\
\hline Win & +100 & +100 & +100 & +100 & +100 & +100 & +100 & +100 & +100 & +100 & +100 & +100 & +100 & +100 & +100 & +100 & +100 & +100 & +100 & +100 \\
\hline Loss & 0 & 0 & 0 & 0 & 0 & 0 & 0 & 0 & $-1,250$ & 0 & 0 & 0 & 0 & $-1,250$ & 0 & 0 & 0 & 0 & 0 & 0 \\
\hline \multicolumn{21}{|l|}{ Deck C } \\
\hline Win & +50 & +50 & +50 & +50 & +50 & +50 & +50 & +50 & +50 & +50 & +50 & +50 & +50 & +50 & +50 & +50 & +50 & +50 & +50 & +50 \\
\hline Loss & 0 & 0 & -50 & 0 & -50 & 0 & -50 & 0 & -50 & -50 & 0 & -25 & -75 & 0 & 0 & 0 & -25 & -75 & 0 & -50 \\
\hline \multicolumn{21}{|l|}{ Deck D } \\
\hline Win & +50 & +50 & +50 & +50 & +50 & +50 & +50 & +50 & +50 & +50 & +50 & +50 & +50 & +50 & +50 & +50 & +50 & +50 & +50 & +50 \\
\hline Loss & 0 & 0 & 0 & 0 & 0 & 0 & 0 & 0 & 0 & -250 & 0 & 0 & 0 & 0 & 0 & 0 & 0 & 0 & 0 & -250 \\
\hline Number of Card & 21 & 22 & 23 & 24 & 25 & 26 & 27 & 28 & 29 & 30 & 31 & 32 & 33 & 34 & 35 & 36 & 37 & 38 & 39 & 40 \\
\hline \multicolumn{21}{|l|}{ Deck A } \\
\hline Win & +100 & +100 & +100 & +100 & +100 & +100 & +100 & +100 & +100 & +100 & +100 & +100 & +100 & +100 & +100 & +100 & +100 & +100 & +100 & +100 \\
\hline Loss & 0 & -300 & 0 & -350 & 0 & -200 & -250 & -150 & 0 & 0 & -350 & -200 & -250 & 0 & 0 & 0 & -150 & -300 & 0 & 0 \\
\hline \multicolumn{21}{|l|}{ Deck B } \\
\hline Win & +100 & +100 & +100 & +100 & +100 & +100 & +100 & +100 & +100 & +100 & +100 & +100 & +100 & +100 & +100 & +100 & +100 & +100 & +100 & +100 \\
\hline Loss & $-1,250$ & 0 & 0 & 0 & 0 & 0 & 0 & 0 & 0 & 0 & $-1,250$ & 0 & 0 & 0 & 0 & 0 & 0 & 0 & 0 & 0 \\
\hline \multicolumn{21}{|l|}{ Deck C } \\
\hline Win & +50 & +50 & +50 & +50 & +50 & +50 & +50 & +50 & +50 & +50 & +50 & +50 & +50 & +50 & +50 & +50 & +50 & +50 & +50 & +50 \\
\hline Loss & 0 & 0 & 0 & -50 & -25 & -50 & 0 & 0 & -75 & -50 & 0 & 0 & 0 & -25 & -25 & 0 & -75 & 0 & -50 & -75 \\
\hline \multicolumn{21}{|l|}{ Deck D } \\
\hline Win & +50 & +50 & +50 & +50 & +50 & +50 & +50 & +50 & +50 & +50 & +50 & +50 & +50 & +50 & +50 & +50 & +50 & +50 & +50 & +50 \\
\hline Loss & 0 & 0 & 0 & 0 & 0 & 0 & 0 & 0 & -250 & 0 & 0 & 0 & 0 & 0 & -250 & 0 & 0 & 0 & 0 & 0 \\
\hline
\end{tabular}


TABLE 2 | Overall win and loss for each IGT deck. Values are in \$.

\begin{tabular}{lcccc}
\hline & Deck A & Deck B & Deck C & Deck D \\
\hline Win & 4,000 & 4,000 & 2000 & 2000 \\
Loss & $-5,000$ & $-5,000$ & $-1,000$ & $-1,000$ \\
Combined & $-1,000$ & $-1,000$ & 1,000 & 1,000
\end{tabular}

principles of the IGT. Subjects now express knowledge about the distribution and the effects of drawing cards from the individual decks.

The total number of advantageous minus disadvantageous selections, i.e., $(C+D)-(A+B)$, typically determines a subject's IGT decision-making (Bechara et al., 1998; Ernst et al., 2003; Franken and Muris, 2005). The more advantageous cards a participant drew, the better their decision-making is. Splitting the results in segments of 20 draws each further allows for an analysis of a subject's selection patterns (Bowman et al., 2005; Bechara et al., 2002; van den Bos et al., 2006). However, due to the dual conception of risk, the IGT results can also be scored according to frequency of preference for immediate reinforcement, i.e., $(B+D)-(A+C)$ (Singh, 2013).

\subsection{Immersive Virtual Reality}

Research already transferred the IGT to VR (Oberdörfer et al., 2020). The study compared the selection patterns between completing the IGT in high immersive $V R$ and in low immersive desktop-3D. Here, the virtual IGT realization integrated the task in a high visual fidelity aquarium environment. The results indicate an impairing effect of higher immersion on IGT decision-making. This result aligns with a similar study showing a negative effect of higher immersion on the risk potential of virtual gambling (Heidrich et al., 2019). In comparison to desktop-3D, wearing a Head-Mounted Display (HMD) increases a user's visual angle on the VE. With a higher visual angle, the emotional responses to audiovisual stimuli are increased (Gall and Latoschik, 2020). According to the Somatic Marker Hypothesis, this would explain the results of the initial IGT VR study. Using the immersive VR version instead of the desktop-3D version increased the visual angle on the IGT. Therefore, it is crucial to further investigate the potentially negative effects of VR. The results might not only be crucial for correctly assessing the risks of virtual gambling scenarios, but also be important for researchers and developers of applications targeting learning and therapy in immersive VR. A negative effect on decision-making caused by the medium might potentially affect the effectiveness, thus requiring specific assistance.

Determining the effect of immersion on human factors of VR systems, e.g., on emotion and cognition (Visch et al., 2010), has a long tradition. Immersion is "the extent to which the computer displays are capable of delivering an inclusive, extensive, surrounding, and vivid illusion of reality to the senses of a human participant" (Slater and Wilbur, 1997). Immersion depends on a system's objective properties reducing real world sensory inputs and replacing them with digital information. This, for instance, is achieved by wearing an HMD. The objective characteristic further describes possible actions within a given system (Slater, 2009). Immersion directly influences presence (Slater et al., 1996; Waltemate et al., 2018). Evoking and maintaining presence requires a continuous stream of stimuli and experience (Witmer and Singer, 1998) as well as a support of sensorimotor contingencies (Slater, 2009). Presence, telepresence, or place illusion describes the subjective sensation of being in a real place despite being physically located in a different place (Slater, 2009). Hence, presence indicates the perceived realness of the virtual experience (Skarbez et al., 2017). In distinction to presence, plausibility illusion describes the subjective illusion of perceiving events inside a VE as real events (Slater, 2009). A high degree of presence positively affects a user's intrinsic motivation for learning (Makransky and Lilleholt, 2018), enhances the overall performance in a training scenario (Stevens and Kincaid, 2015) especially when a high visual fidelity is provided (McMahan et al., 2012; Ragan et al., 2015), and increases the emotions experienced in a VE (Riva et al., 2007).

\subsection{Emotional Effects of Virtual Environments}

External stimuli and their individual perception can evoke and determine emotions (Plutchik, 1982; Ellsworth and Smith, 1988). From an evolutionary point of view, emotions prepare the human body to react to dangers and to social situations (Plutchik, 1982; Lynch and Martins, 2015). Even awareness of virtuality cannot suppress these instincts (Baird, 2000). Hence, the human brain involuntarily reacts to virtual stimuli, e.g., the design of a VE, with emotion and behavior (Reeves and Nass, 1997).

Aside from concrete events, the surrounding environment is a prominent external stimulus affecting a person's mood. Research demonstrates that people feel happier outdoors. Ranked by their impact, people prefer to be in 1) sea and coastal regions, 2) mountains, moors, heathlands, 3) forests, and 4) semi-natural grassland (MacKerron and Mourato, 2013). The desire for access to nature also affects the design of everyday places. Cities benefit from various types of vegetation that "create an atmosphere of lushness, green and shade" (Botkin, 1997). Providing access to nature, e.g., window views, plants, and landscapes, leads to a lower stress and higher job satisfaction in working environments and a better recovery of patients in hospitals (Ulrich, 2001; Dijkstra et al., 2006; Dinis et al., 2013). Daytime environments are appraised as significantly more pleasant than dark places (Toet et al., 2009). Considering the light, people perceive warm reddish light as pleasant, thus experiencing positive feelings such as enthusiasm and joy (Baron et al., 1992; Knez and Niedenthal, 2008). In contrast, a lack of windows in closed rooms leads to anxiety and depression (Ulrich, 2001). The feeling of being trapped induces feelings of isolation, uncertainty, and anxiety (Steinmetz, 2018). With regard to illumination, dimly lit environments evoke fear due to the lack of visual information (Grillon et al., 1997; Niedenthal, 2007). Also, people feel less relaxed and less pleased in cooly-lit rooms (Baron et al., 1992).

These positive and negative effects of environmental characteristics create the two extremes for the design space of 

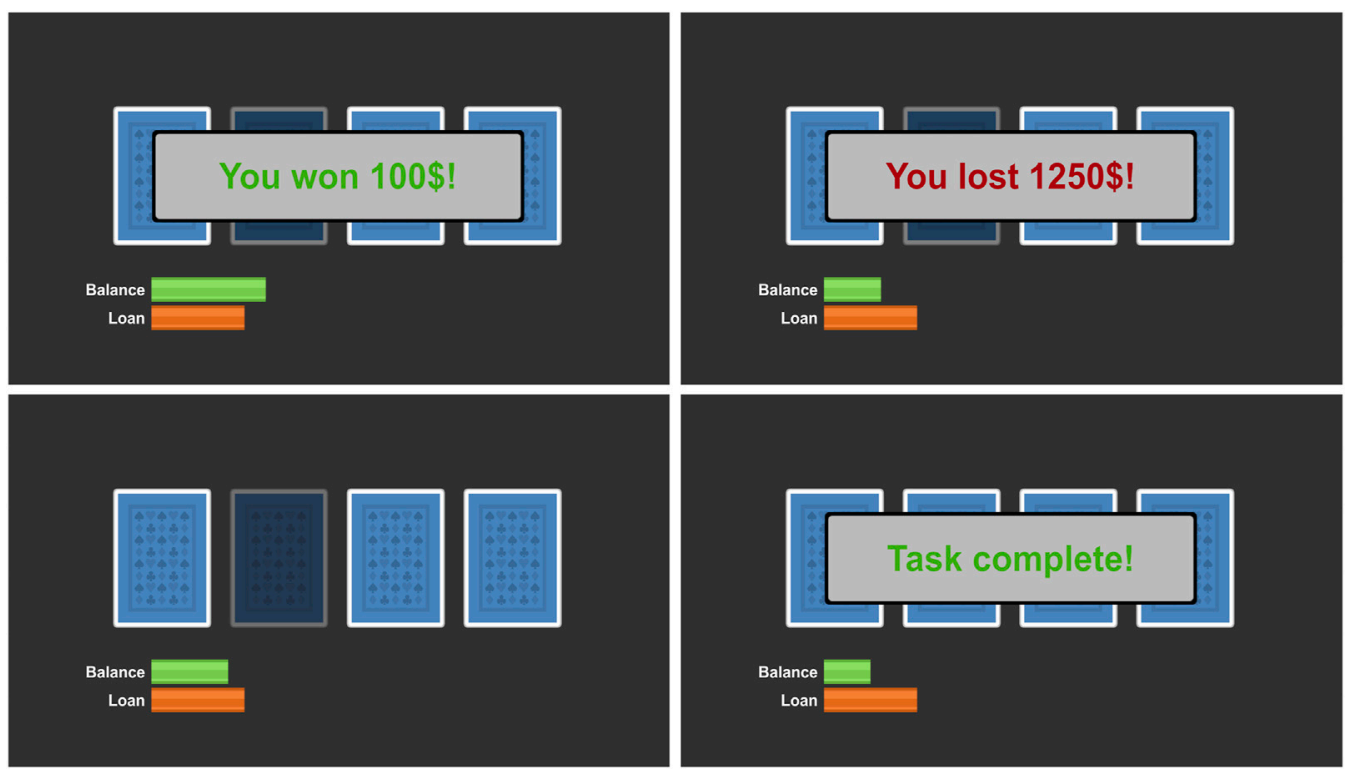

FIGURE 2 | Decks are displayed lying face down. The orange bar indicates a player's loan and the green bar represents the current balance. Top left: The player's win is displayed for $2 \mathrm{~s}$. Top right: The loss is shown for $2 \mathrm{~s}$. Bottom left: A player drew all cards from a deck. Bottom right: The player drew 100 cards and finished the task.

VEs. By designing VEs within the margin of these boundaries, either positive or negative emotions can be evoked and supported in a user.

\subsection{Summary}

The IGT measures decision-making under the circumstance of an uncertainty in the context of punishment and reward. When completed in immersive VR, subjects showed an impaired decision-making in contrast to a desktop-3D condition. Since decision-making can be influenced by emotions, the higher visual angle on the VE might explain this effect. The design of VEs can further affect a user's emotional state. Hence, administrating the IGT in different VEs might lead to different selection patterns and decision-making results.

\section{SYSTEM DESCRIPTION}

To analyze the effects of a higher immersion without an increased visual angle on the task and VE design on decision-making, we developed three IGT applications: a low immersion Desktop, a high immersion VR Desktop, and a high immersion VR Forest version. While all versions implement the same IGT realization, they differ in the medium used and environment. The Desktop version displays the virtual IGT on a physical computer screen. The VR Desktop version replicates the physical laboratory as a 3D-model-based VE. It displays the virtual IGT on a 3D model of the physical computer screen. The virtual computer screen has the same dimensions as the physical computer screen. The $V R$ Forest version uses a 3D-model-based VE of a forest featuring a wooden cabin. On the cabin's porch, we placed the 3D model of the computer screen on a wooden table to show the IGT gameplay. With regard to emotional VE design, Desktop as well as VR Desktop represent the negative side of the design space and VR Forest follows the theoretical best characteristics. We kept the position of the user relative to the computer screen the same across all versions. This ensured the same visual angle on the IGT and the surrounding environment.

\subsection{Virtual lowa Gambling Task}

Our virtual IGT encodes the fixed schedule of win and loss for each card of the original IGT version as shown in Table 1. Upon drawing a card, the respective deck's payout is first added to the player's balance, and the card's loss value is then subtracted from the player's balance. Our virtual IGT plays a short sound-effect to provide audiovisual feedback and displays the four card decks lying face down. Additionally, the system displays the player's current balance and initial loan with two labeled bars. A green bar indicates the player's balance and an orange bar indicates the initial loan. The virtual IGT adjusts the length of the balance bar according to the win and loss of a game round. Simultaneously, the system displays the payout and, in the case of a loss, also the lost money as displayed in Figure 2. The information is displayed for $2 \mathrm{~s}$. During this payout phase, a player cannot draw a new card. After drawing 40 cards from a single deck, the respective deck becomes inactive. The virtual IGT informs the player about the completion of the task after the 100th card is drawn.

Our realization of the virtual IGT features two core interactions: 1) selection of a deck and 2) drawing a card from the selected deck as depicted in Figure 3. To ensure for a comparability between the three versions, the interactions and the user interface (UI) need to be the same for all versions. We selected the HTC Vive Pro (HTC Corporation, 2011-2021) as the output device for the VR version. Research demonstrated a better 


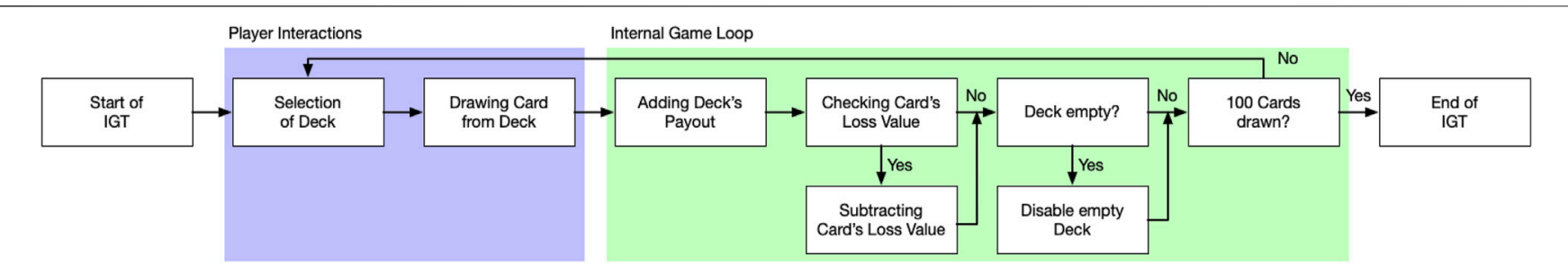

FIGURE 3 | Visualization of the IGT game loop. After selecting a deck, players can draw a card. The IGT checks the player's selection, executes the payout process and checks if the deck is empty as well as if the IGT is complete.
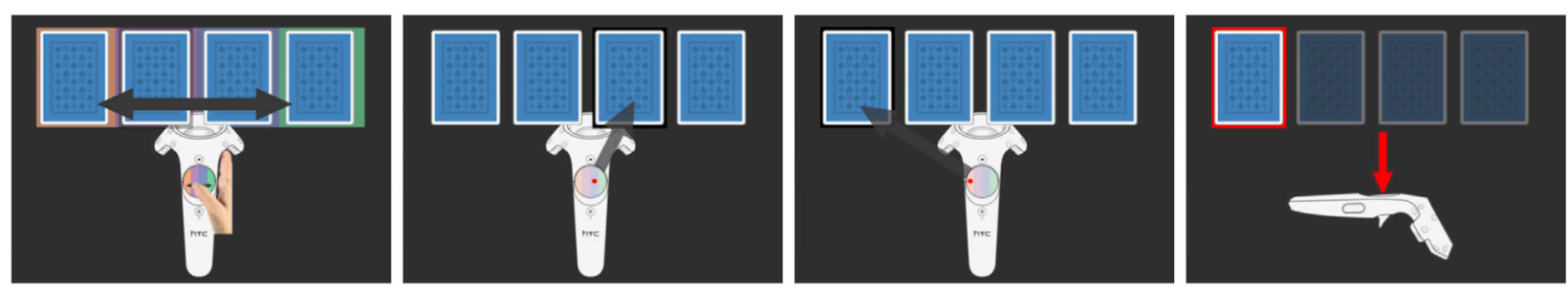

FIGURE 4 | Visualization of the IGT selection technique. Each card deck has a respective zone on the controller's touchpad. By swiping the finger over the sensor, a user selects a deck. The user draws a card by pressing down the touchpad.

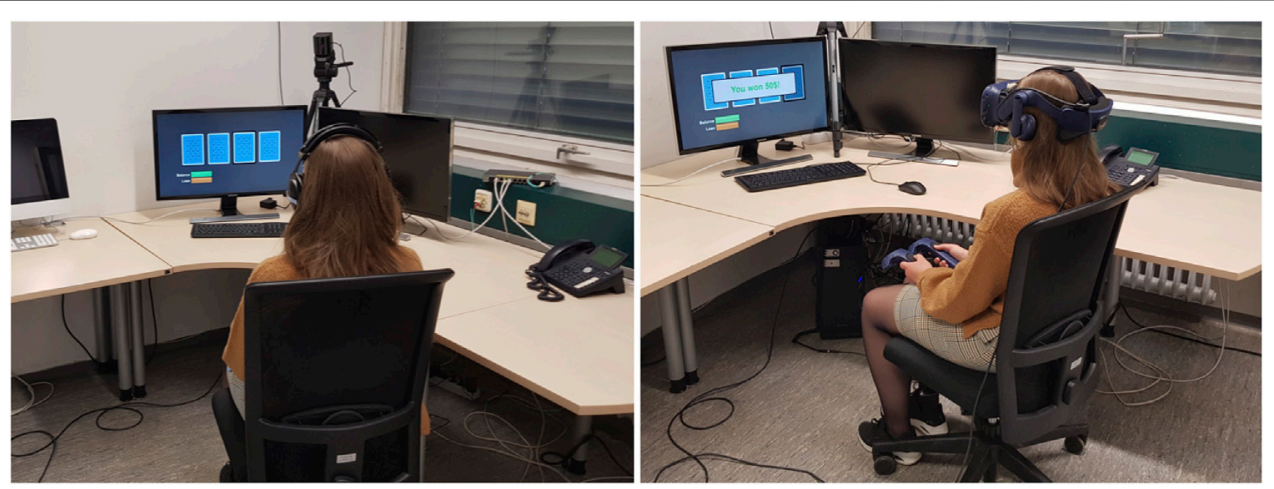

FIGURE 5 | We matched the position of the virtual screens with the physical screen. In all conditions, participants sat at a distance of $1.3 \mathrm{~m}$ away from the screen. Left: Participant plays the desktop version. Right: Participant completes a VR version of the IGT.

performance when requiring subjects to first draw a physical card before making the same selection in the computerized version of the IGT in contrast to only drawing cards in the computerized IGT (Vanhille et al., 2018). The same study revealed a better performance for touch interactions on a tablet in comparison to clicking with a mouse. However, the results only show that the type of task administration yields different results, but causes no negative effect on the validity of virtual-only versions of the IGT. Hence, we implemented a single HTC Vive game controller as the main input device for the virtual IGT. Using the controller's touchpad, a player can physically and directly select a deck and draw a card from it as displayed in Figure 4. Since the IGT features four decks, we defined four zones on the touchpad. A player selects a deck by merely touching a specific zone or moving the finger over the touchpad until the desired zone is activated. A black frame marks the currently selected deck. Pressing the touchpad draws a card from the deck. The controller is physically and visually present when playing the desktop version. Therefore, we implemented it as a diegetic UI element in the VR versions by using its 3D model as Figure 5 displays (LaViola et al., 2017).

We developed the virtual IGT with Unity 2019.3.10f1 (Unity, 2021) using the SteamVR plugin version 1.2.3 (Valve Coorperation, 2015-2021). The virtual IGT is a prefab allowing for a free positioning inside of VEs.

\subsection{Environmental Design}

For playing the desktop condition, we used a computer setup in one of our laboratories. The lab consists of a large corner desk with three monitors and a chair as shown in Figure 1. 
Participants played the IGT on the computer screen in the center. Following the characteristics of emotionally negative environment design, we shut the blinds of the windows and illuminated the room with ceiling lights. This resulted in the laboratory being a cooly-lit and closed environment without access to nature. In addition, this setup ensured the same environmental conditions throughout a day. The door of the room is located on the opposite side of the windows. Next to it, we positioned a small desk and a chair for the experimenter. For the $V R$ Desktop version, we created an exact 3D-model-based VE of the laboratory as displayed in Figure 1. This laboratory VE also elicits the negative aspects of emotional environment design.

In contrast, VR Forest is based on the theoretical best characteristics of a positive emotional design as discussed in Section 2.3. We created a forest VE featuring a pleasant atmosphere to induce positive emotions in the participants. In detail, we designed a landscape of hills covered with conifers and bushes to create a pleasant view with different vegetation. We decided to use a brightly lit daylight setting with a few light clouds in the sky. To increase the plausibility of interacting with the IGT in the forest, we placed a wooden cabin in the VE. We further placed a rustic style wooden table and chair on the cabin's terrace as shown in Figure 1. For showing the gameplay of the IGT, we positioned the same virtual computer screen as in the VR Desktop on the wooden table. To lighten up the VE, we positioned colorful bright flowers in the participants' field of view. We created the VE with Unity using prefab assets.

\section{METHODS}

Based on our theoretical considerations in Section 2 and the design of the three IGT versions described in Section 3, we assume the following hypotheses:

$\mathrm{H} 1$ : Higher immersion causes no significant impairment of decision-making when the visual angle on the task is kept the same.

$\mathrm{H} 2$ : An emotionally positive environmental design evokes a significantly higher emotional state in a user during the completion of the IGT.

$\mathrm{H} 3$ : An emotionally positive environmental design causes a better IGT decision-making.

To test our hypotheses, we compared the three versions of the IGT in a user study with respect to IGT decision-making, presence, mood, and task load. When decision-making is unimpaired, participants develop an understanding for the structure of the IGT over the course of the experiment (Bechara et al., 1997, 2005). As a result, participants no longer deal with uncertainties when repeatedly completing the IGT. Therefore, we chose a between subjects experimental design. We randomly assigned the participants to one of the three conditions, i.e., Desktop condition, VR Desktop condition, or VR Forest condition. However, we balanced the conditions with respect to prior VR experience of the participants. As described in section 3 , the three versions differ in the medium used and environment. Thus, the independent variables were the degree of immersion, i.e., low immersion desktop condition and high immersion VR condition, and environment, i.e., laboratory and forest. We only used participants who reported no signs of gambling addiction.

Our study was approved by the Human-Computer-Media institutional ethics review board of the University of Würzburg.

\subsection{Apparatus}

The experimental setup consisted of a desk, a chair, a computer (CPU: i7-9700K, RAM: 16GB, GPU: RTX 2070), one 28 inches computer screen (resolution: $3840 \times 2160 \mathrm{px}$ ), an HTC Vive Pro HMD $\left(1440 \times 1600 \mathrm{px}\right.$ resolution per eye, $110^{\circ}$ field of view $)$, a single HTC Vive controller, a mouse, and a keyboard. Participants sat at the desk throughout the entire experimental session. While filling in the questionnaires, they could position the chair as they wanted. For playing the IGT, we asked them to position the chair at a specific location: $1.3 \mathrm{~m}$ away from the computer screen. Since we matched the position of the virtual screens in the VR Desktop and VR Forest version with the position of the physical computer screen, this ensured the same visual angle on the IGT gameplay across all conditions. The participants used over-ear headphones for playing the Desktop version. For the two VR versions, participants used the HTC Vive HMD headphones.

The experimenter was required to remain in the room for safety reasons. Research by Rockloff and Greer (2011); Molde et al. (2017) demonstrates that the presence of an observer can lead to less risky gambling behavior. In light of this, the experimenter could have confounded the study. Thus, to limit this potential confounding effect, we positioned the experimenter's desk out of the participant's line of sight and away from the center of the room. Also, the experimenter pretended to work during the playing session.

\subsection{Measures}

For evaluating our virtual IGT versions and testing our hypotheses, we used the following measures.

\subsubsection{Demographics}

For demographic data, we assessed a participant's age (in years), gender, video game experience (hours per week), VR experience (hours total), and gambling attitude ( $1=$ dislike, $5=$ like). As an additional control variable, the pre-questionnaire included the Immersive Tendency Questionnaire (ITQ) (Witmer and Singer, 1998) to assess a participant's immersive tendency, their current alertness as well as fitness, and their ability to focus.

\subsubsection{Simulator Sickness}

We measured the simulator sickness for all participants before and after the simulation using the Simulator Sickness Questionnaire (SSQ) (Kennedy et al., 1993). The SSQ scales range from 0 to 3 . The total scores were calculated as described by Kennedy et al. (1993). Low scores stand for low simulator sickness.

\subsubsection{Decision-Making}

We used our virtual IGT to measure decision-making. As described in section 2.1, the IGT requires subjects to draw 100 cards from four decks which are either advantageous or 
disadvantageous. The total number of advantageous minus disadvantageous selections determines a subject's IGT decision-making (Bechara et al., 1998; Ernst et al., 2003; Franken and Muris, 2005). A higher number of advantageous cards drawn indicates a better IGT decision-making.

\subsubsection{Decision Time}

The virtual IGT versions further logged the decision time (seconds) for each game round. We measured this value as a control variable to check whether the participants were influenced by the design of a VE. The change in decision time can reflect the development of explicit knowledge about the IGT (Cella et al., 2007). Thus, analyzing this variable can provide further insights into the IGT decision-making.

\subsubsection{Presence}

The study included the presence questionnaire-version 3.0 (PQ) consisting of the 19 core items (Witmer et al., 2005). The PQ consists of 7-point Likert scales $(7=$ high perceived presence).

\subsubsection{Task Load}

The NASA Task Load Index (NASA-TLX) (Hart and Staveland, 1988) measures the perceived task load. To facilitate the evaluation process, we used the Raw NASA-TLX (Hart, 2006). It eliminates the weighting process and only includes the six subscales (Moroney et al., 1992). We calculated the score for each subscale as described by Hart and Staveland (1988) leading to total scores ranging from 0 to 100 . Low scores mean low task load and high performance. We administered the NASA-TLX as a control variable to check whether the requirement to wear the HMD or the design of the VEs affected the perceived task load.

\subsubsection{Positive Affect and Negative Affect}

For measuring the mood, we use the Positive and Negative Affect Schedule (PANAS) (Watson and Clark, 1988). The PANAS consists of two 10-item 5-point Likert scales (5 = very much). Each scale measures one of the two primary dimensions of mood, i.e., positive and negative effects. As we wanted to determine the overall effect of the VE design, we only measured the mood at the end of the experiment.

\subsection{Procedure}

The study took place during the Covid-19 pandemic. To ensure for protection and hygiene, we took the following precautions. 1) Each participant was required to disinfect their hands before and after the study, constantly wear a mask, and report whether they stayed in a risk area or show signs of an illness. 2) The experimenter was required to disinfect their hands, constantly wear a mask, and daily report whether they show signs of an illness. 3) The experimenter and the participant were required to keep at least a distance of $1.5 \mathrm{~m}$ 4) All touched surfaces and used devices, e.g., HMD, controllers, keyboard, had to be cleaned with a disinfectant product after each experimental trial. 5) The laboratory had to be ventilated for at least 15 min after each experimental trial.

After being welcomed, the experimenter told the participant to sit down at the desk, to read the study information, and to sign an informed consent form. Each participant had to fill in the
Problem Gambling Severity Index (PGSI) (Caler et al., 2016). We administered the PGSI as a safety measurement to protect them against gambling related harm. This 9-item questionnaire measures the severity of a gambling addiction by considering a person's gambling behavior over the past year (Ferris and Wynne, 2001). We only allowed participants that scored 0 on the PGSI to take part in the experiment. Afterwards, participants filled in the pre-questionnaire consisting of the demographics questionnaire, ITQ, and SSQ. At the end of the pre-questionnaire, the participants received written and illustrated instructions about the IGT gameplay. Here, we also used the images of Figure 4 to explain the card selection interaction technique. Subsequently, the experimenter instructed the participants to position their chairs at the correct location. The participants completed the IGT in the randomly assigned version. In the case of a VR condition, we also informed them about the functionality of the HMD and the symptoms of cybersickness. After completing the IGT, the participants filled in the post-questionnaire consisting of the PQ, SSQ, NASA-TLX, and PANAS. Finally, we explained the goal of the experiment as well as the IGT's fixed schedule of win and loss, showed a short educational video about problem gambling, and thanked the participants. In the case of a VR condition, we reminded them of the effects of cybersickness. Figure 6 provides an overview of our procedure.

\subsection{Participants}

We recruited participants from the staff and students enrolled at the University of Würzburg. Participants belonging to the group of students were rewarded with credits mandatory for obtaining their program of study's degrees. In total, 60 participants took part in the study. Table 3 provides an overview of the participants' demographic data. 48 participants reported a previous VR experience $(M=62.53 h, S D=120.14 h)$ and 46 participants reported to play video games for $M=4.95 \mathrm{~h}$ per week $(S D=6.02 h)$. None of them had completed the IGT before.

\section{RESULTS}

For comparing the results, we computed an analysis of variance (ANOVA). We used a repeated measures analysis of variance (RM-ANOVA) to analyze the decision-making patterns over the course of the task. Effect sizes were determined by computing $\eta^{2}$. We used Pearson's $r$ to check for correlations. Table 4 gives an overview of the descriptive statistics.

\subsection{Simulator Sickness}

We found no significant difference between the two times of measurement for the SSQ for the VR Desktop, $t(19)=3.02, p=$ 0.78 , and the VR Forest, $t(19)=0.17, p=0.87$, conditions. However, in the Desktop condition the simulator sickness was significantly lower after the stimulus with a medium effect size, $t$ $(19)=3.01, p<0.01, d=0.68$.

\subsection{Decision-Making}

We computed and analyzed the traditional and intertemporal IGT scores, i.e., $(C+D)-(A+B)$ (Bechara et al., 1998; Ernst et al., 2003; 


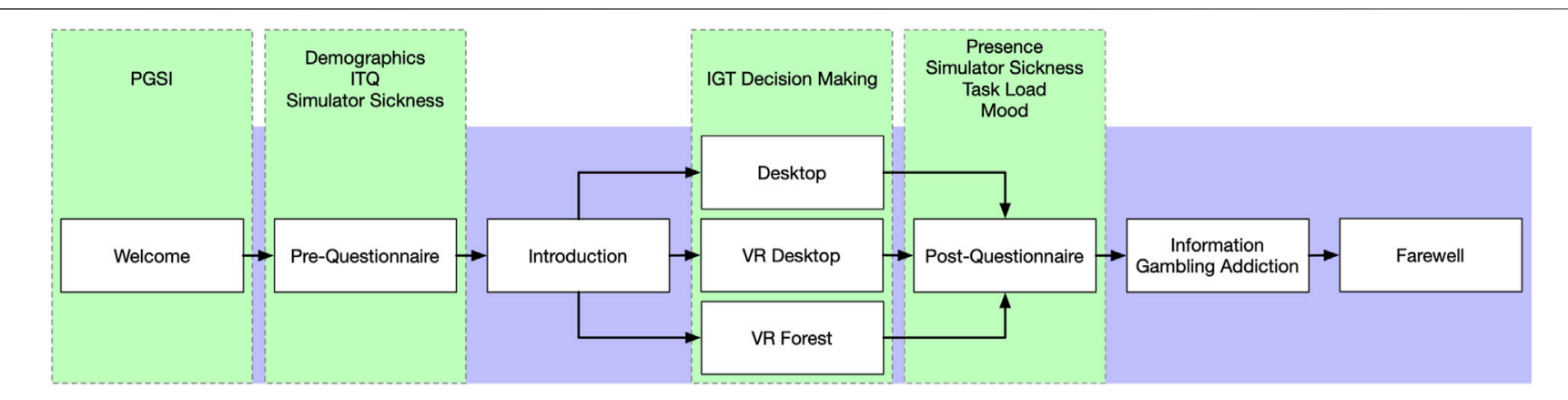

FIGURE 6 | Overview of the experimental procedure. The figure combines the individual steps with the measurements used.

TABLE 3 | Demographic data. Values are either $M(S D)$ or $N(\%)$.

\begin{tabular}{|c|c|c|c|}
\hline & Desktop & VR desktop & VR forest \\
\hline & $N=20$ & $N=20$ & $N=20$ \\
\hline Age & $23.85(4.23)$ & $23.25(4.44)$ & $23.25(2.69)$ \\
\hline Males & $8(40 \%)$ & $6(30 \%)$ & $8(40 \%)$ \\
\hline Females & $12(60 \%)$ & $14(70 \%)$ & $12(60 \%)$ \\
\hline VR experience (number of participants) & $16(80 \%)$ & $17(85 \%)$ & $15(75 \%)$ \\
\hline VR experience (hours/week) & $65.36(128.06)$ & $33.79(73.56)$ & $92.07(150.85)$ \\
\hline Gaming experience (number of participants) & $18(90 \%)$ & $14(70 \%)$ & $14(70 \%)$ \\
\hline Gaming experience (hours/week) & $5.39(5.55)$ & $3.65(3.94)$ & $5.70(8.18)$ \\
\hline Gambling attitude & $2.45(0.95)$ & $2.40(0.94)$ & $2.10(0.85)$ \\
\hline ITQ & $4.39(0.56)$ & $4.58(0.44)$ & $4.38(0.57)$ \\
\hline
\end{tabular}

TABLE 4 | Descriptive statistics. Values are $M(S D)$.

\begin{tabular}{|c|c|c|c|}
\hline & Desktop & VR desktop & VR forest \\
\hline & $N=20$ & $N=20$ & $N=20$ \\
\hline SSQ-pre & $7.85(6.75)$ & $13.09(14.18)$ & $14.96(25.31)$ \\
\hline SSQ-post & $4.49(5.23)$ & $13.65(11.97)$ & $14.40(16.69)$ \\
\hline IGT (intertemporal) & $-5.90(25.07)$ & $4.60(21.30)$ & $-0.90(23.82)$ \\
\hline IGT (frequency of reinforcement) & $5.70(13.49)$ & $9.20(12.25)$ & $10.00(15.83)$ \\
\hline $\mathbf{P Q}$ & $3.55(0.73)$ & $4.14(0.89)$ & $3.69(0.54)$ \\
\hline Task load & $21.00(13.25)$ & $21.79(11.91)$ & $25.29(12.23)$ \\
\hline Positive affect & $20.80(6.32)$ & $20.20(5.75)$ & $21.45(5.53)$ \\
\hline Negative affect & $20.25(5.11)$ & $19.70(4.77)$ & $21.30(5.08)$ \\
\hline
\end{tabular}

Franken and Muris, 2005). We found no significant difference between the three conditions with respect to IGT decisionmaking, $F(2,57)=1.00, p=0.37, \eta^{2}=0.03$; see Figure 7 left. Splitting the intertemporal IGT decision-making in segments of 20 draws, we found no significant effect of the condition on the decision-making patterns, $F(6.55,186.79)=1.14, p=0.34, \eta^{2}=$ 0.02; see Figure 8, using a Greenhouse-Geisser correction.

Due to the dual conception of risk, the IGT results can also be scored according to frequency of preference for immediate reinforcement, i.e., $(B+D)-(A+C)$ (Singh, 2013). We found no significant difference between the conditions with respect to the frequency of reinforcement IGT decision-making, $F(2,57)=$ $0.54, p=0.59, \eta^{2}=0.02$; see Figure 7 right. Splitting the frequency of reinforcement IGT decision-making in segments of 20 draws, we found no significant effect of the condition on the decision- making patterns, $F(6.49,184.91)=0.72, p=0.64, \eta^{2}=0.02$, using a Greenhouse-Geisser correction.

We found no correlation between intertemporal and frequency of reinforcement IGT decision-making for any demographic property, decision time, presence, task load, and positive as well as negative affect.

\subsection{Decision Time}

As displayed in Figure 9, the decision time strongly declined in all conditions during the first two phases. This reflects the emotional learning effect (Cella et al., 2007). However, splitting the IGT decision-time in segments of 20 draws, we found no significant effect of the condition on the decision time, $F$ $(2.87,81.84)=1.83, p=0.15, \eta^{2}=0.02$, using a GreenhouseGeisser correction. 

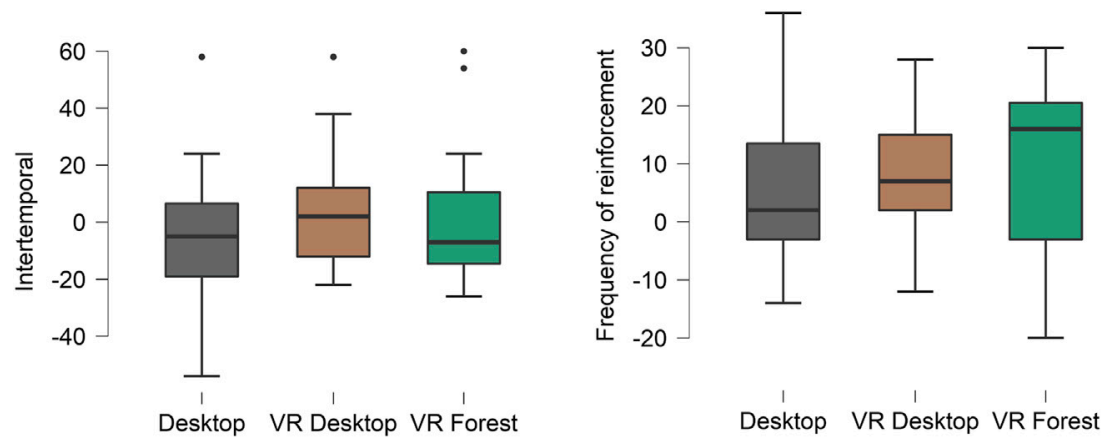

FIGURE 7 | Tukey-style box plots for the traditional intertemporal (left) and frequency of reinforcement (right) IGT decision-making scores. The median (line within the box), first and third quartiles (box), non-outlier range (whiskers), and outliers (dot) are shown.

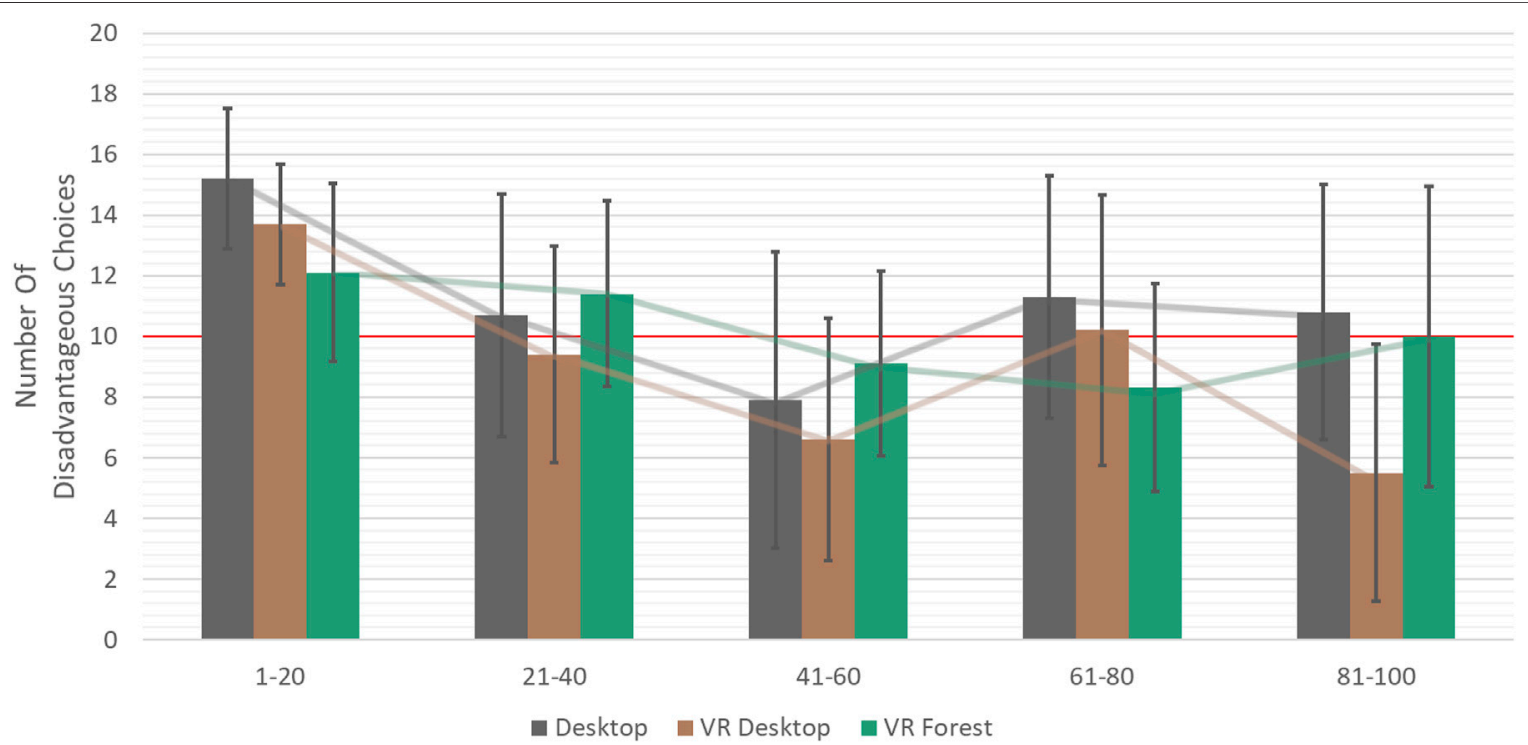

FIGURE 8 | Comparison of the mean number of disadvantageous cards drawn per 20 game rounds. The error bars denote the standard deviation. The red line indicates the threshold above which more bad than good decisions were made.

\subsection{Presence}

We did find a significant difference between the three conditions with respect to presence, $F(2,57)=3.54, p=0.04, \eta^{2}=0.11$; see Figure 10 middle left. Post hoc tests with a Tukey correction revealed a significant difference between the conditions Desktop and VR Desktop, $t(59)=-2.55, p=0.04, d=-0.73$.

\subsection{Task Load}

We found no significant difference between the three conditions with respect to task load, $F(2,57)=0.67, p=0.52, \eta^{2}=0.02$.

\subsection{Positive Affect and Negative Affect}

We found no significant difference between the three conditions with respect to positive affect, $F(2,57)=0.23, p=0.80, \eta^{2}=0.01$; see Figure 10 middle right, and to negative affect, $F(2,57)=0.53$, $p=0.59, \eta^{2}=0.02$; see Figure 10 right.

\section{DISCUSSION}

We found no significant difference between the two measurements of the SSQ. Neither VR condition induced simulator sickness. Overall, the SSQ-post scores were very low across all conditions. However, we found a significant difference for the desktop condition. Here, the SSQ ratings were significantly higher before the experimental trial. This phenomenon could be an effect of excitement or uncertainty. Alternatively, participants could have rushed to the experiment to arrive on time. These effects declined over the course of the experiment and led to a significantly lower SSQ rating.

As expected, presence was rated higher in both VR conditions in comparison to the desktop condition. Surprisingly, we only found a significant difference between Desktop and VR Desktop but not between Desktop and VR Forest. This result is explainable 

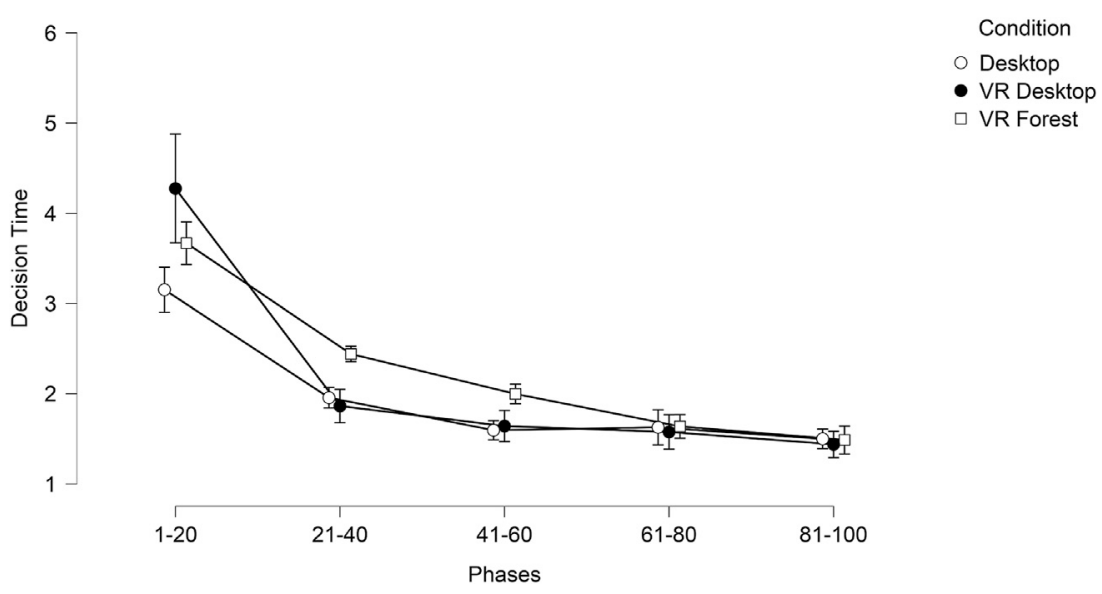

FIGURE 9 | Comparison of the mean decision time per 20 game rounds. The error bars denote the standard error.
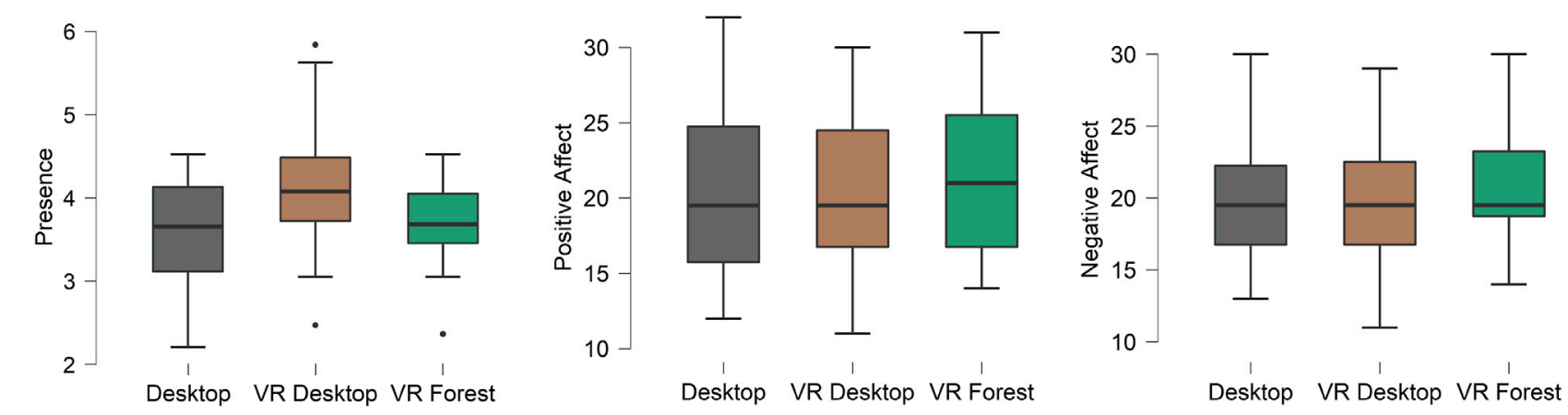

FIGURE 10 | Tukey-style box plots for the ratings of presence, positive affect, and negative affect. The median (line within the box), first and third quartiles (box), non-outlier range (whiskers), and outliers (dot) are shown.

by a higher prediction accuracy of haptic information in $V R$ Desktop than in VR Forest. A higher prediction accuracy leads to increased presence ratings, whereas a low prediction accuracy decreases presence ratings (Gall and Latoschik, 2018). As we matched the positions of the desk and the computer screens between Desktop and VR Desktop, we yielded coherent haptic information in our laboratory VE. The wooden table in the forest VE did not match the dimensions of the physical desk. This might have influenced the presence ratings in the VR Forest condition. A different explanation or potentially contributing factor might be a slightly higher visual fidelity of the laboratory VE in comparison to the forest VE. A higher visual fidelity increases the experienced presence (McMahan et al., 2012; Ragan et al., 2015). We created all assets for the laboratory VE ourselves. This resulted in an almost photorealistic VE design and hence in a higher presence in comparison to the forest VE.

Task load did not differ significantly between the conditions. This indicates that the requirement to wear the HMD caused no negative side-effect. It further indicates that the design of the tested VEs did not moderate the perception of the task load.

\subsection{IGT Decision-Making}

Our study revealed no significant difference between the conditions neither with respect to intertemporal nor frequency of reinforcement IGT decision-making (see Figure 10 left). As shown in Figure 8, the conditions did not differ significantly with regard to the 20-card selection patterns. Finally, the decision-making time remained unaffected by the three virtual IGT versions. This strongly suggests that neither immersion nor the design of our two VEs causes an impairment of decision-making.

H1 supported: Since we kept the visual angle on the IGT the same by either using a physical or virtual computer screen, we only manipulated the degree of immersion. Despite the change in immersion, IGT decision-making did not differ significantly. Hence, there are no indications that immersion causes impairment of decision-making as long as the visual angle on the stimulus remains the same.

As discussed in section 2.1 and section 2.2, this result is explainable by the Somatic Marker Hypothesis. Emotions affect decision-making. An increased visual angle on a stimulus can lead 
to a higher emotional response. We used the same visual angle on the IGT in all conditions. Hence, the emotional response evoked by the visual angle on the stimulus was the same between the conditions. Analyzing the results of the PANAS revealed no significant difference between the conditions. This backs our assumption and is especially relevant for the comparison of the Desktop and VR Desktop versions. These two versions only differ with respect to immersion. Thus, by keeping the emotional state constant, our results suggest no effect of impairment of decision-making caused by immersion.

H2 rejected: We found no significant difference in IGT decision-making caused by the design of the VEs. Despite our efforts of providing the theoretically best characteristics of a positive emotional VE design in VR Forest and of a negative design in Desktop and VR Desktop, the tested VEs did not differ significantly with regard to positive or negative affect. This especially is relevant for the comparison of the VR Desktop and VR Forest versions. These two versions only differ with regard to the design of the $\mathrm{VE}$.

This outcome could be a result of the presentation of the IGT. We chose to present the IGT on a virtual computer screen of the same dimensions as the physical one. In this way, the gameplay took place in only a very small portion of the entire VE. Like in the physical world, participants had to focus on the virtual computer screen. Hence, the surrounding VE was not as perceptible as when the IGT would have been presented more prominently. A different explanation might be the structure of the task. We told the participants to play the game. As a result, they might have felt the urge to immediately start playing without taking in the atmosphere of the VE. Finally, the result could be explained by a strong effect of flow (Csikszentmihalyi, 1990; McGonigal, 2011). Evoked by the clear goal and the immediate feedback, participants might have been in a strong state of flow. Thus, they fully focussed their attention on the IGT gameplay and blended out all other stimuli, i.e., the VE.

The lack of a significant difference with regard to the PANAS results between the two VEs could also be an indication that the visual differences were not strong enough. A real-world forest provides a different scent, temperature, sound, and overall atmosphere that goes beyond the visual illusion of being in a forest. Despite these limitations, our two VEs should still facilitate an investigation into the effect evoked by the VE design in our first experiment. Here, we used an aquarium VE that created the visual illusion of being underwater without providing any other stimuli. Similar to the forest VR, the aquarium also elicited some characteristics of a positive emotional design, e.g., a daytime environment featuring warm colors.

H3 rejected: The tested VEs did not influence the participants' emotional state. Thus, they caused no effect on IGT decisionmaking as explained by the Somatic Marker Hypothesis. As a result, being immersed in the two tested VEs causes no impairment of decision-making.

\subsection{Limitations}

The study took place during the Covid-19 pandemic. New governmental measures regarding a lockdown went into effect over the course of the experiment. Despite our measurements for protection and hygiene, these events might have subconsciously affected the participants. The unconscious effects potentially influenced the emotional state of the participants. The unconscious effects further might have reduced the positive and negative affect evoked by the design of the VEs.

Aside from the Covid-19 pandemic, the measurements for protection and hygiene potentially affected our results even further. A few participants reported fogging of the HMD lenses caused by the requirement of wearing a mask. While not totally obscuring their view, the fogging might have limited the perception of the surrounding VE. As a result, the differences between the VR Desktop and VR Forest conditions might have been mostly blocked out. The participants saw the IGT gameplay on the virtual computer screen, but not the VE itself. The fogging further reduced the field of view of the HMD and hence reduced the immersion. This potentially could have affected the perceived presence of the participants.

Our tested VEs only differed with regard to the overall environmental characteristics to evoke either positive or negative emotions. Considering the overall research goal of investigating the risk potential of gambling in VR, this leaves out gambling related aspects such as visual cues disguising losses as wins (Graydon et al., 2017) or music (Griffiths and Parke, 2005). Although these elements also belong to the VE design, we purposefully focussed on the overall environmental characteristics since they are independent of the specific structure of a gambling game. However, it would be an interesting research direction to also investigate the influence of specific gambling aspects on IGT decision-making.

Lastly, our study neglected a potential influence of other prominent VR factors. Our virtual IGT could be altered by providing an embodiment of the participant (IJsselsteijn et al., 2006; Slater et al., 2009; Lugrin et al., 2015). The experience of an illusion of virtual body ownership increases presence (Waltemate et al., 2018). Research by Riva et al. (2007) demonstrates that presence increases experienced emotions. As a result of this, IGT decision-making may be affected. Embodiment further can evoke the Proteus effect (Yee and Bailenson, 2007). Depending on the avatar appearance (Latoschik et al., 2016; Roth et al., 2016), providing an embodiment could also influence the IGT.

\section{Implications}

In combination with our previous study's results (Oberdörfer et al., 2020), these findings are notable. The first IGT VR study found a significant difference between a desktop and a VR version of the IGT with regard to decision-making. The two versions directly embedded the IGT in a VE. As a result, the VR version provided a higher visual angle on the task. Thus, we hypothesized that the higher visual angle was the true cause for the measured difference. Our results of the present study test this hypothesis, thus extending the findings of the first experiment. We investigated whether immersion causes an impairment of decision-making when the visual angle remains unchanged. We further evaluated the effects of different VE designs. Since neither immersion nor design of the VEs caused an impairment of decision-making, our results provide first indications that the higher visual angle on the IGT in VR potentially causes the 
significant difference in decision-making. To validate our result, future work should focus on a comparison of different visual angles on the IGT in the same VE. With such an experimental design, the effect of the visual angle on IGT decision-making can be analyzed.

Taken together, our results lead to the following implications. 1) We demonstrate that high visual immersion does not affect IGT decision-making as long as the visual angle on the task is kept the same. As a result, 2) using immersive VR can safely be used for decision-making training in critical situations as well as for therapeutic applications. 3) We further recommend that the visual angle on a game should be considered when assessing the overall risk potential of a VR gambling game. Research already demonstrates a higher risk potential of gambling games when played in immersive VR (Heidrich et al., 2019).

\section{CONCLUSION}

This article reported novel findings on IGT decision-making in immersive VR. Our contributions are twofold. We 1) investigated the effects of immersion and design of VEs on decision-making and 2) compared our results to our first study of administrating the IGT in VR. We kept the visual angle on the IGT the same across all conditions.

\subsection{Findings}

We found no significant impairment of decision-making caused by the degree of immersion or the design of the VEs. Furthermore, our conditions did not differ significantly with regard to positive and negative affect. Combined with the findings of our previous IGT VR experiment (Oberdörfer et al., 2020), our results provide first indications for a moderating effect of the visual angle on IGT decision-making. A higher visual angle increases emotional responses to stimuli (Gall and Latoschik, 2020) and hence potentially directly affects decision-making. Additionally, for assessing the overall risk of an immersive VR gambling game, we recommend that the visual angle on the game is considered.

\subsection{Future Work}

Future work should focus a comparison of varied visual angles on the IGT. Such an experiment could validate whether the visual angle truly effects IGT decision-making. A different research

\section{REFERENCES}

Armstrong, T., Rockloff, M., Greer, N., and Donaldson, P. (2017). Rise of the Machines: A Critical Review on the Behavioural Effects of Automating Traditional Gambling Games. J. Gambl Stud. 33, 735-767. doi:10.1007/ s10899-016-9644-4

Baird, R. (2000). The Startle Effect: Implications for Spectator Cognition and Media Theory. Film Q. 53, 12-24. doi:10.1525/fq.2000.53.3.04a00030

Baron, R. A., Rea, M. S., and Daniels, S. G. (1992). Effects of Indoor Lighting (Illuminance and Spectral Distribution) on the Performance of Cognitive Tasks and Interpersonal Behaviors: The Potential Mediating Role of Positive Affect. Motiv. Emot. 16, 1-33. doi:10.1007/BF00996485 direction should be to investigate whether other prominent VR factors influence decision-making. For instance, using an embodied VE and manipulating the avatar appearance could have an effect on IGT decision-making in immersive VR.

\section{DATA AVAILABILITY STATEMENT}

The raw data supporting the conclusion of this article will be made available by the authors, without undue reservation.

\section{ETHICS STATEMENT}

The studies involving human participants were reviewed and approved by Human-Computer-Media institutional ethics review board of the University of Würzburg. The patients/participants provided their written informed consent to participate in this study.

\section{AUTHOR CONTRIBUTIONS}

$\mathrm{SO}, \mathrm{DH}$, and $\mathrm{ML}$ contributed equally to the conception of this research. DH developed the IGT VR system and the cardselection technique. SB designed the two VEs and created the assets used in the laboratory VE. SO, DH, and SB designed as well as conducted the user study, and analyzed the data. $\mathrm{SO}, \mathrm{DH}$, and SB wrote the manuscript.

\section{FUNDING}

This publication was supported by the Open Access Publication Fund of the University of Würzburg.

\section{ACKNOWLEDGMENTS}

We would like to thank Kilian Röder for his support in conducting the user study. Also, we would like to thank Sophia C. Steinhaeusser for the discussion about the emotional design of VEs.

Bechara, A., Damasio, A. R., Damasio, H., and Anderson, S. W. (1994). Insensitivity to Future Consequences Following Damage to Human Prefrontal Cortex. Cognition. 50, 7-15. doi:10.1016/0010-0277(94)90018-3

Bechara, A., and Damasio, A. R. (2005). The Somatic Marker Hypothesis: A Neural Theory of Economic Decision. Games Econ. Behav. 52, 336-372. doi:10.1016/ j.geb.2004.06.010

Bechara, A., Damasio, H., Tranel, D., and Anderson, S. W. (1998). Dissociation of Working Memory From Decision Making Within the Human Prefrontal Cortex. J. Neurosci. 18, 428-437. doi:10.1523/ jneurosci.18-01-00428.1998

Bechara, A., Damasio, H., Tranel, D., and Damasio, A. R. (1997). Deciding Advantageously Before Knowing the Advantageous Strategy. Science. 275, 1293-1295. doi:10.1126/science.275.5304.1293 
Bechara, A., Damasio, H., Tranel, D., and Damasio, A. R. (2005). The Iowa Gambling Task and the Somatic Marker Hypothesis: Some Questions and Answers. Trends Cogn. Sci. 9, 159-162. doi:10.1016/ j.tics.2005.02.002

Bechara, A., Dolan, S., and Hindes, A. (2002). Decision-Making and Addiction (Part Ii): Myopia for the Future or Hypersensitivity to Reward?. Neuropsychologia. 40, 1690-1705. doi:10.1016/s0028-3932(02)00016-7

Bechara, A., Tranel, D., and Damasio, H. (2000). Characterization of the DecisionMaking Deficit of Patients With Ventromedial Prefrontal Cortex Lesions. Brain. 123, 2189-2202. doi:10.1093/brain/123.11.2189

Betz, L. T., Brambilla, P., Ilankovic, A., Premkumar, P., Kim, M.-S., Raffard, S., et al. (2019). Deciphering Reward-Based Decision-Making in Schizophrenia: A Meta-Analysis and Behavioral Modeling of the Iowa Gambling Task. Schizophrenia Res. 204, 7-15. doi:10.1016/j.schres.2018.09.009

Botkin, D. B., and Beveridge, C. E. (1997). Cities as Environments. Urban Ecosyst. 1, 3-19. doi:10.1023/a:1014354923367

Bowman, C. H., Evans, C. E. Y., and Turnbull, O. H. (2005). Artificial Time Constraints on the Iowa Gambling Task: The Effects on Behavioural Performance and Subjective Experience. Brain Cogn. 57, 21-25. doi:10.1016/ j.bandc.2004.08.015

Brevers, D., Bechara, A., Cleeremans, A., and Noël, X. (2013). Iowa Gambling Task (IGT): Twenty Years after - Gambling Disorder and IGT. Front. Psychol. 4, 665. doi:10.3389/fpsyg.2013.00665

Brevers, D., Cleeremans, A., Goudriaan, A. E., Bechara, A., Kornreich, C., Verbanck, P., et al. (2012). Decision Making Under Ambiguity but Not under Risk Is Related to Problem Gambling Severity. Psychiatry Res. 200, 568-574. doi:10.1016/j.psychres.2012.03.053

Caler, K., Garcia, J. R. V., and Nower, L. (2016). Assessing Problem Gambling: a Review of Classic and Specialized Measures. Curr. Addict. Rep. 3, 437-444. doi:10.1007/s40429-016-0118-7

Cauffman, E., Shulman, E. P., Steinberg, L., Claus, E., Banich, M. T., Graham, S., et al. (2010). Age Differences in Affective Decision Making as Indexed by Performance on the Iowa Gambling Task. Developmental Psychol. 46, 193-207. doi:10.1037/a0016128

Cella, M., Dymond, S., Cooper, A., and Turnbull, O. (2007). Effects of DecisionPhase Time Constraints on Emotion-Based Learning in the Iowa Gambling Task. Brain Cogn. 64, 164-169. doi:10.1016/j.bandc.2007.02.003

Chiu, Y.-C., Huang, J.-T., Duann, J.-R., and Lin, C.-H. (2018). Editorial: Twenty Years After the Iowa Gambling Task: Rationality, Emotion, and DecisionMaking. Front. Psychol. 8, 2353. doi:10.3389/fpsyg.2017.02353

Csikszentmihalyi, M. (1990). Flow: The Psychology of Optimal Experience. New York, NY, USA: Harper-Perennial.

Damasio, A. R., Tranel, D., and Damasio, H. C. (1991). "Somatic markers and the guidance of behavior: Theory and preliminary testing," in Frontal lobe function and dysfunction. Editors H. S. Levin, H. M. Eisenberg, and A. L. Benton (Oxford, UK: Oxford University Press), 217-229.

de Vries, M., Holland, R. W., and Witteman, C. L. M. (2008). In the Winning Mood: Affect in the Iowa Gambling Task. Judgment Decis. Making. 3, 42-50.

DeDonno, M. A., and Demaree, H. A. (2008). Perceived Time Pressure and the Iowa Gambling Task. Judgment Decis. making. 3, 636-640.

Dijkstra, K., Pieterse, M., and Pruyn, A. (2006). Physical Environmental Stimuli that Turn Healthcare Facilities Into Healing Environments Through Psychologically Mediated Effects: Systematic Review. J. Adv. Nurs. 56, 166-181. doi:10.1111/j.1365-2648.2006.03990.x

Dinis, S., Duarte, E., Noriega, P., Teixeira, L., Vilar, E., and Rebelo, F. (2013). "Evaluating Emotional Responses to the interior Design of a Hospital Room: A Study Using Virtual Reality," in International Conference of Design, User Experience, and Usability (Springer), 475-483. doi:10.1007/978-3-64239238-2_52

Dunn, B. D., Dalgleish, T., and Lawrence, A. D. (2006). The Somatic Marker Hypothesis: A Critical Evaluation. Neurosci. Biobehavioral Rev. 30, 239-271. doi:10.1016/j.neubiorev.2005.07.001

Ellsworth, P. C., and Smith, C. A. (1988). Shades of joy: Patterns of Appraisal Differentiating Pleasant Emotions. Cogn. Emot. 2, 301-331. doi:10.1080/ 02699938808412702

Ernst, M., Grant, S. J., London, E. D., Contoreggi, C. S., Kimes, A. S., and Spurgeon, L. (2003). Decision Making in Adolescents with Behavior Disorders and Adults With Substance Abuse. Ajp. 160, 33-40. doi:10.1176/appi.ajp.160.1.33
Ferris, J. A., and Wynne, H. J. (2001). The Canadian Problem Gambling index. Ottawa: Canadian Centre on Substance Abuse Ottawa, ON.

Flores-Torres, J., Gómez-Pérez, L., McRae, K., López, V., Rubio, I., and Rodríguez, E. (2019). Humor Improves Women's but Impairs Men's Iowa Gambling Task Performance. Front. Psychol. 10, 2538. doi:10.3389/fpsyg.2019.02538

Franken, I. H. A., and Muris, P. (2005). Individual Differences in Decision-Making. Personal. Individual Differences. 39, 991-998. doi:10.1016/j.paid.2005.04.004

Gainsbury, S. M., Suhonen, N., and Saastamoinen, J. (2014). Chasing Losses in Online Poker and Casino Games: Characteristics and Game Play of Internet Gamblers at Risk of Disordered Gambling. Psychiatry Res. 217, 220-225. doi:10.1016/j.psychres.2014.03.033

Gall, D., and Latoschik, M. E. (2018). "The Effect of Haptic Prediction Accuracy on Presence," in Proceedings of the 25th IEEE Virtual Reality conference (VR '18), 73-80. doi:10.1109/vr.2018.8446153

Gall, D., and Latoschik, M. E. (2020). Visual Angle Modulates Affective Responses to Audiovisual Stimuli. Comput. Hum. Behav. 109, 106346. doi:10.1016/ j.chb.2020.106346

Graydon, C., Dixon, M. J., Harrigan, K. A., Fugelsang, J. A., and Jarick, M. (2017). Losses Disguised as Wins in Multiline Slots: Using an Educational Animation to Reduce Erroneous Win Overestimates. Int. Gambling Stud. 17, 442-458. doi:10.1080/14459795.2017.1355404

Griffiths, M. (2017). The Psychosocial Impact of Gambling in Virtual Reality. Casino Gaming Int. 29, 51-54.

Griffiths, M., and Parke, J. (2005). The Psychology of Music in Gambling Environments: An Observational Research Note. J. Gambling Issues (Jgi)., 1-12. doi:10.4309/jgi.2005.13.8

Grillon, C., Pellowski, M., Merikangas, K. R., and Davis, M. (1997). Darkness Facilitates the Acoustic Startle Reflex in Humans. Biol. Psychiatry. 42, 453-460. doi:10.1016/S0006-3223(96)00466-0

Hart, S. G. (2006). "Nasa-task Load index (Nasa-tlx); 20 Years Later," in Proceedings of the Human Factors and Ergonomics Society 50th Annual Meeting (Los Angeles, CA: Santa Monica), 50, 904-908. doi:10.1177/ 154193120605000909

Hart, S. G., and Staveland, L. E. (1988). "Development of Nasa-Tlx (Task Load index): Results of Empirical and Theoretical Research," in Human Mental Workload. Editors P. A. Hancock and N. Meshkati (Amsterdam: Elsevier Science Publishers B.V.), 139-183. doi:10.1016/s0166-4115(08)62386-9

Heidrich, D., Oberdörfer, S., and Latoschik, M. E. (2019). "The Effects of Immersion on Harm-Inducing Factors in Virtual Slot Machines," in Proceedings of the 26th IEEE Virtual Reality conference (VR '19) (Osaka, Japan: IEEE). doi:10.1109/vr.2019.8798021

Heilman, R. M., Crişan, L. G., Houser, D., Miclea, M., and Miu, A. C. (2010). Emotion Regulation and Decision Making under Risk and Uncertainty. Emotion. 10, 257-265. doi:10.1037/a0018489

[Dataset] HTC Corporation (2011-2021). Htc Vive. available at https://www.vive. com (Accessed July 19, 2021).

IJsselsteijn, W. A., de Kort, Y. A. W., and Haans, A. (2006). Is This My Hand I See before Me? the Rubber Hand Illusion in Reality, Virtual Reality, and Mixed Reality. Presence: Teleoperators and Virtual Environments. 15, 455-464. doi:10.1162/pres.15.4.455

İyilikci, E. A., and Amado, S. (2018). The Uncertainty Appraisal Enhances the Prominent Deck B Effect in the Iowa Gambling Task. Motiv. Emot. 42, 1-16. doi:10.1007/s11031-017-9643-5

Kahneman, D., and Frederick, S. (2007). Frames and Brains: Elicitation and Control of Response Tendencies. Trends Cogn. Sci. 11, 45-46. doi:10.1016/ j.tics.2006.11.007

Kennedy, R. S., Lane, N. E., Berbaum, K. S., and Lilienthal, M. G. (1993). Simulator Sickness Questionnaire: An Enhanced Method for Quantifying Simulator Sickness. Int. J. Aviation Psychol. 3, 203-220. doi:10.1207/s15327108ijap0303_3

Knez, I., and Niedenthal, S. (2008). Lighting in Digital Game Worlds: Effects on Affect and Play Performance. CyberPsychology Behav. 11, 129-137. doi:10.1089/cpb.2007.0006

Latoschik, M. E., Lugrin, J.-L., and Roth, D. (2016). "FakeMi: A Fake Mirror System for Avatar Embodiment Studies," in Proceeding of ACM Symposium on Virtual Reality Software and Technology.

LaViola, J. J., Jr, Kruijff, E., McMahan, R. P., Bowman, D., and Poupyrev, I. P. (2017). 3D User Interfaces: Theory and Practice. Boston, MA: Addison-Wesley Professional. 
Leder, J., Horlitz, T., Puschmann, P., Wittstock, V., and Schütz, A. (2019). Comparing Immersive Virtual Reality and Powerpoint as Methods for Delivering Safety Training: Impacts on Risk Perception, Learning, and Decision Making. Saf. Sci. 111, 271-286. doi:10.1016/j.ssci.2018.07.021

Lee, W.-K., Su, Y.-A., Song, T.-J., Chiu, Y.-C., and Lin, C.-H. (2014). Are normal Decision-Makers Sensitive to Changes in Value Contrast Under Uncertainty? Evidence From the Iowa Gambling Task. Plos One. 9, e101878-10. doi:10.1371/ journal.pone. 0101878

Lugrin, J.-L., Latt, J., and Latoschik, M. E. (2015). "Anthropomorphism and Illusion of Virtual Body Ownership," in Proceedings of the 25th International Conference on Artificial Reality and Telexistence and 20th Eurographics Symposium on Virtual Environments, 1-8.

Lynch, T., and Martins, N. (2015). Nothing to Fear? an Analysis of College Students' Fear Experiences With Video Games. J. Broadcasting Electron. Media. 59, 298-317. doi:10.1080/08838151.2015.1029128

MacKerron, G., and Mourato, S. (2013). Happiness Is Greater in Natural Environments. Glob. Environ. Change. 23, 992-1000. doi:10.1016/ j.gloenvcha.2013.03.010

Maia, T. V., and McClelland, J. L. (2004). A Reexamination of the Evidence for the Somatic Marker Hypothesis: What Participants Really Know in the Iowa Gambling Task. Proc. Natl. Acad. Sci. 101, 16075-16080. doi:10.1073/ pnas.0406666101

Makransky, G., and Lilleholt, L. (2018). A Structural Equation Modeling Investigation of the Emotional Value of Immersive Virtual Reality in Education. Educ. Technology Res. Development. 66, 1141-1164. doi:10.1007/ s11423-018-9581-2

McGonigal, J. (2011). Reality Is Broken: Why Games Make Us Better and How They Can Change the World. 1st edn. New York: Penguin Press.

McMahan, R. P., Bowman, D. A., Zielinski, D. J., and Brady, R. B. (2012). Evaluating Display Fidelity and Interaction Fidelity in a Virtual Reality Game. IEEE Trans. Vis. Comput. Graphics. 18, 626-633. doi:10.1109/tvcg.2012.43

Miu, A. C., Heilman, R. M., and Houser, D. (2008). Anxiety Impairs DecisionMaking: Psychophysiological Evidence From an Iowa Gambling Task. Biol. Psychol. 77, 353-358. doi:10.1016/j.biopsycho.2007.11.010

Molde, H., Mentzoni, R., Hanss, D., Sagoe, D., Andersen, S. L., and Pallesen, S. (2017). People Around You - Do They Matter? an Experimental Gambling Study. Int. Gambling Stud. 17, 349-365. doi:10.1080/14459795.2017.1333130

Moroney, W. F., Biers, D. W., Eggemeier, F. T., and Mitchell, J. A. (1992). "A Comparison of Two Scoring Procedures With the Nasa Task Load index in a Simulated Flight Task," in Proceedings of the IEEE 1992 National Aerospace and Electronics Conference (NAECON), 734-740.

Niedenthal, S. (2007). "Shadowplay: Simulated Illumination in Game Worlds," in Worlds in Play. Editors S. Niedenthal, S. d. Castell, and J. Jenson (New York, NY: Lang: New literacies and digital epistemologies).

Oberdörfer, S., Heidrich, D., and Latoschik, M. E. (2020). "Think Twice: The Influence of Immersion on Decision Making during Gambling in Virtual Reality," in Proceedings of the 27th IEEE Virtual Reality conference (VR '20) (Atlanta, USA: IEEE), 483-492. doi:10.1109/VR46266.2020.00069

Oberdörfer, S., and Latoschik, M. E. (2013). "Develop Your Strengths by Gaming: Towards an Inventory of Gamificationable Skills," in Informatik 2013 Informatik angepasst an Mensch, Organisation und Umwelt. Editor M. Horbach (Koblenz, Germany: Gesellschaft für Informatik e.V.), 2346-2357.

Oberdörfer, S., and Latoschik, M. E. (2018). "Gamified Knowledge Encoding: Knowledge Training Using Game Mechanics," in Proceedings of the 10th International Conference on Virtual Worlds and Games for Serious Applications (VS Games 2018) Würzburg, Germany. doi:10.1109/vsgames.2018.8493425

Pittig, A., Brand, M., Pawlikowski, M., and Alpers, G. W. (2014). The Cost of Fear: Avoidant Decision Making in a Spider Gambling Task. J. Anxiety Disord. 28, 326-334. doi:10.1016/j.janxdis.2014.03.001

Plutchik, R. (1982). A Psychoevolutionary Theory of Emotions. Soc. Sci. Inf. 21, 529-553. doi:10.1177/053901882021004003

Preston, S. D., Buchanan, T. W., Stansfield, R. B., and Bechara, A. (2007). Effects of Anticipatory Stress on Decision Making in a Gambling Task. Behav. Neurosci. 121, 257-263. doi:10.1037/0735-7044.121.2.257

Ragan, E. D., Bowman, D. A., Kopper, R., Stinson, C., Scerbo, S., and McMahan, R. P. (2015). Effects of Field of View and Visual Complexity on Virtual Reality
Training Effectiveness for a Visual Scanning Task. IEEE Trans. Vis. Comput. Graphics. 21, 794-807. doi:10.1109/tvcg.2015.2403312

Reeves, B., and Nass, C. I. (1997). The media Equation: How People Treat Computers, Televisions, and New media like Real People and Places. 2nd printing edn. Stanford, California: CSLI Publ.

Riva, G., Mantovani, F., Capideville, C. S., Preziosa, A., Morganti, F., Villani, D., et al. (2007). Affective Interactions Using Virtual Reality: the Link between Presence and Emotions. CyberPsychology Behav. 10, 45-56. doi:10.1089/cpb.2006.9993

Rockloff, M. J., and Greer, N. (2011). Audience Influence on Egm Gambling: The Protective Effects of Having Others Watch You Play. J. Gambl Stud. 27, 443-451. doi:10.1007/s10899-010-9213-1

Roth, D., Lugrin, J.-L., Galakhov, D., Hofmann, A., Bente, G., Latoschik, M. E., et al. (2016). "Avatar Realism and Social Interaction Quality in Virtual Reality," in Proceedings of IEEE Virtual Reality, 277-278. doi:10.1109/vr.2016.7504761

Singh, V. (2013). Dual conception of Risk in the Iowa Gambling Task: Effects of Sleep Deprivation and Test-Retest gap. Front. Psychol. 4, 628. doi:10.3389/ fpsyg.2013.00628

Singh, V., and Khan, A. (2008). Heterogeneity in Choices on iowa Gambling Task: Preference for Infrequent-High Magnitude Punishment. Mind Soc. 8, 43. doi:10.1007/s11299-008-0050-1

Skarbez, R., Brooks, Jr., F. P., Jr., and Whitton, M. C. (2018). A Survey of Presence and Related Concepts. ACM Comput. Surv. 50, 1-39. doi:10.1145/3134301

Slater, M., Linakis, V., Usoh, M., and Kooper, R. (1996). Immersion, Presence, and Performance in Virtual Environments: An experiment with Tri-dimensional Chess. ACM Virtual Reality Softw. Technology (Vrst)., 163-172. doi:10.1145/ 3304181.3304216

Slater, M., Perez-Marcos, D., Ehrsson, H. H., and Sanchez-Vives, M. V. (2009). Inducing Illusory Ownership of a Virtual Body. Front. Neurosci. 3, 214-220. doi:10.3389/neuro.01.029.2009

Slater, M. (2009). Place Illusion and Plausibility Can lead to Realistic Behaviour in Immersive Virtual Environments. Phil. Trans. R. Soc. B. 364, 3549-3557. doi:10.1098/rstb.2009.0138

Slater, M., and Wilbur, S. (1997). A Framework for Immersive Virtual Environments (Five): Speculations on the Role of Presence in Virtual Environments. Presence: Teleoperators \& Virtual Environments. 6, 603-616. doi:10.1162/pres.1997.6.6.603

Steinmetz, K. F. (2018). Carceral Horror: Punishment and Control in Silent Hill. Crime, Media, Cult. 14, 265-287. doi:10.1177/1741659017699045

Stevens, J. A., and Kincaid, J. P. (2015). The Relationship between Presence and Performance in Virtual Simulation Training. OJMSi. 03, 41-48. doi:10.4236/ ojmsi.2015.32005

Toet, A., van Welie, M., and Houtkamp, J. (2009). Is a Dark Virtual Environment Scary?. CyberPsychology Behav. 12, 363-371. doi:10.1089/cpb.2008.0293

Ulrich, R. S. (2001). "Effects of Healthcare Environmental Design on Medical Outcomes," in Design and Health: Proceedings of the Second International Conference on Health and Design (Stockholm, Sweden: Svensk Byggtjanst), 59.49

[Dataset] Unity (2021). unity 2019.3.10f1. Available at: https://unity3d.com/getunity/download/archive. (Accessed July 19, 2021).

[Dataset] Valve Coorperation (2015-2021). Steamvr Plugin. Available at: https:// www.assetstore.unity3d.com/en/\\#!/content/32647. (Accessed July 19, 2021).

van den Bos, R., Homberg, J., and de Visser, L. (2013). A Critical Review of Sex Differences in Decision-Making Tasks: Focus on the iowa Gambling Task. Behav. Brain Res. 238, 95-108. doi:10.1016/j.bbr.2012.10.002

van den Bos, R., Houx, B. B., and Spruijt, B. M. (2006). The Effect of Reward Magnitude Differences on Choosing Disadvantageous Decks in the Iowa Gambling Task. Biol. Psychol. 71, 155-161. doi:10.1016/j.biopsycho.2005.05.003

Vanhille, S., Palmer, B., Hayes, W., and Overman, W. (2018). The Effect of Active Participation on Performance and Understanding on the iowa Gambling Task. J. Behav. Dec Making. 31, 686-694. doi:10.1002/bdm.2083

Verdejo-Garcia, A., Benbrook, A., Funderburk, F., David, P., Cadet, J.-L., and Bolla, K. I. (2007). The Differential Relationship between Cocaine Use and Marijuana Use on Decision-Making Performance Over Repeat Testing With the Iowa Gambling Task. Drug and alcohol dependence. 90, 2-11. doi:10.1016/ j.drugalcdep.2007.02.004

Visch, V. T., Tan, E. S., and Molenaar, D. (2010). The Emotional and Cognitive Effect of Immersion in Film Viewing. Cogn. Emot. 24, 1439-1445. doi:10.1080/ 02699930903498186 
Waltemate, T., Gall, D., Roth, D., Botsch, M., and Latoschik, M. E. (2018). The Impact of Avatar Personalization and Immersion on Virtual Body Ownership, Presence, and Emotional Response. IEEE Trans. Vis. Comput. Graphics. 24, 1643-1652. doi:10.1109/tvcg.2018.2794629

Watson, D., Clark, L. A., and Tellegen, A. (1988). Development and Validation of Brief Measures of Positive and Negative Affect: The Panas Scales. J. Personal. Soc. Psychol. 54, 1063-1070. doi:10.1037/0022-3514.54.6.1063

Witmer, B. G., Jerome, C. J., and Singer, M. J. (2005). The Factor Structure of the Presence Questionnaire. Presence: Teleoperators \& Virtual Environments. 14, 298-312. doi:10.1162/105474605323384654

Witmer, B. G., and Singer, M. J. (1998). Measuring Presence in Virtual Environments: A Presence Questionnaire. Presence. 7, 225-240. doi:10.1162/ 105474698565686

Yee, N., and Bailenson, J. (2007). The Proteus Effect: The Effect of Transformed Self-Representation on Behavior. Hum. Comm Res. 33, 271-290. doi:10.1111/ j.1468-2958.2007.00299.x
Conflict of Interest: The authors declare that the research was conducted in the absence of any commercial or financial relationships that could be construed as a potential conflict of interest.

Publisher's Note: All claims expressed in this article are solely those of the authors and do not necessarily represent those of their affiliated organizations, or those of the publisher, the editors and the reviewers. Any product that may be evaluated in this article, or claim that may be made by its manufacturer, is not guaranteed or endorsed by the publisher.

Copyright (c) 2021 Oberdörfer, Heidrich, Birnstiel and Latoschik. This is an open-access article distributed under the terms of the Creative Commons Attribution License (CC BY). The use, distribution or reproduction in other forums is permitted, provided the original author(s) and the copyright owner(s) are credited and that the original publication in this journal is cited, in accordance with accepted academic practice. No use, distribution or reproduction is permitted which does not comply with these terms. 